\title{
Sexual Dysfunction as a Side Effect of Hyperprolactinemia in Methadone Maintenance Therapy
}

\author{
Aneta Spasovska Trajanovska'1, Viktorija Vujovic², Liljana Ignjatova1', Danijela Janicevic-Ivanovska ${ }^{3}$, Andon Cibisev ${ }^{4}$ \\ Day hospital for drug addiction, Psychiatric hospital Skopje, R. Macedonia ${ }^{1}$ University Psychiatric Clinic, Medical Faculty , \\ Skopje, R. Macedonia² University Biochemistry Clinic, Medical faculty, Skopje, R. Macedonia ${ }^{3}$ University Clinic of toxicology, \\ Medical Faculty Skopje, R. Macedonia ${ }^{4}$
}

A lthough endocrine abnormalities are recognized in opiate users, very little is known about the range of hormones affected, their pathophysiology and their clinical relevance. Various endocrine abnormalities have been reported in these patients including, increased prolactin levels and abnormalities in sexual hormone. Path physiological mechanism postulated does explain these findings including direct action of heroin or methadone at the hypothalamic pituitary level. The aim of this study was to explore the effects of heroin and methadone maintenance treatment on the plasma prolactin levels and sexual function. Material and methods: We evaluated 20 male narcotic addicts maintained of methadone more than 3 years on oral high dose methadone 60-120 mgr/day. Patients taking neuroleptic therapy were excluded from the study because neuroleptic-included hyperprolactinemia. We also evaluated group of twenty male heroin addicts on the street heroin. The prolactin plasma levels were assayed using the chemiluminescent immunometric essay (CLIA) - high sensitive methods, The normal range of prolactin levels was 1,5-17 ng/ml(53-360 nmol/l) for men and 1,90-25,0 $\mathrm{ng} / \mathrm{ml}$ for women. The sexual function was assessed using a Questionnaire: International Index of Erectile Function (IIEF) with 15 items in four levels of sexual function. The differences between two examination groups were determined by a student's $t$ test. The results show that street heroin addicts ( $55 \%$ of them have high level of prolactin) have significantly higher plasma prolactin levels $(\mathrm{p}=0,006)$ then the group of methadone maintenance patients (only 15\% of them have high prolactin level). In our study, when we compared sexual dysfunction in examination groups in some domains, we did not find statistical significant results (sexual desire $\mathrm{p}=0,52$ and overall satisfaction $\mathrm{p}=0,087$ ). But in domains of erectile function $\mathrm{p}=0,011$ and orgasm function $\mathrm{p}=0,033$ we got statistical significant results. Key words: heroin addiction, methadone maintenance therapy, sexual dysfunction, prolactin plasma levels.

Corresponding author: Aneta Spasovska Trajanovska, MD. Day hospital for drug addiction, Psychiatric hospital Skopje, R Macedonia

\section{INTRODUCTION}

Although endocrine abnormalities are recognized in opiate users in literature research very little is known about the range of hormones affected, their pathophysiology, and their clinical relevance. Various endocrine abnormalities have been reported in these patients, which included: increased levels of thyroxine (T4), done at the hypothalamic or pituitary levels. Some literature research conclude that many of the hypothalamic and anterior pituitary hormones are adversely affected in heroin misuses and methadone maintained patients (1). But, one of the most seriously endocrine abnormalities in opiate users and methadone maintenance patients is hyperprolactinemia and sexual dysfunction as a side-effects of opioid induced hyperprolactinemia (2). Prolactin is synthesized and secretion by lacto trope cells in adenophypophysis (anterior pituitary gland). Pituitary prolactin secretion is regulated by neuroendocrine neurons in the hypothalamus, the most important ones being the neurosecretory tuberoinfundibulum (TIDA) neurons of the arcuate nucleus with secrete dopamine to act on the dopamine -2 receptors (D2-R) of lactotrophs causing inhibition of prolactin secretion. So, dopamine has an inhibitory effect on prolactin excretion (3, 4). Pathophysiological mechanism postulated does explain these findings included a direct action of heroin or methadone at the hypothalamic or pituitary level. In all parts of the brain, opioids stimulate dopamine production engendering analgesia, euphoria, and other reward effects of opioids. Only in the hypothalamus opioids decreasing dopamine secretion, it produces excess amounts of prolactin a condition influencing sexual dysfunction called hyperprolactinemia (5). Hyperprolactinemia acts to gonad function included reproductive dys- 
function (an ovulation, fertility. Decreased estrogen and testosterone production) and sexual dysfunction (sexual impairment, erectile dysfunction, retrograde or painful ejaculation, orgasmic dysfunction) (6, 7). However some research study reported that steady-state pharmacokinetics properties of methadone allow adaptation and normalization of endocrine and neuroendocrine function. So optimum methadone doses tend to stabilize hormonal balances over time (6).

The AIM of this study was to explore the effects of heroin and methadone maintenance therapy on the plasma prolactin levels and sexual function in men.

\section{MATERIAL AND METHODS}

The present study included two groups of heroin addicts. The first group A included 20 male heroin addicts who was on the street heroin. The second B group consisted of twenty male heroin addicts who were on the methadone maintenance therapy more than 3 years, on oral high dose methadone 60-120 mgr/day. After complete description on the study, a written informed consent was obtained. Confidentially of information was assured. The study protocol was approved by the local ethics commitment and is consistent with the Helsinki declaration. All the patients followed criteria for opioid dependence and had no other significant psychiatric diagnosis. Especially patients with neuroleptic therapy were excluded from the study because neuroleptic therapy induced hyperprolactinemia. Criteria to elimination was: chronic liver or renal diseases or other chronic physical disorders, HIV diseases or active infection. All the patients there followed by routine medical examination, with included blood chemistry, urine analysis and serology for hepatitis and HIV infection. Hormone assays: blood samples of all patients and heroin street addicts were obtained the morning between 09.00 $11.00 \mathrm{H}$. The prolactin plasma levels were assayed using the chemiluminescent immunometric assay (CLIA)-high sensitive methods. The normal range of prolactin level was $1,5-17,0 \mathrm{ng} / \mathrm{ml}$ for men and 1,90-25,0 $\mathrm{ng} / \mathrm{ml}$ for women. Sexual function in males was assessed by using the Questionnaire (IIEF) which contains 15 items. All the items are scored in 4 domains (erectile function, orgasmic function, sexual desire, overall satisfaction). Final scores can be interpreted as following score: severe, moderate, mild to moderate, mild, and without dysfunction. The differences of hormone levels and scores of sexual dysfunction among the groups were compared by using the t-test for independent samples.

\section{RESULTS}

Hormone analysis in our study show that 55 percent of heroin addicts have high plasma prolactin levels and $45 \%$ of them have normal prolactin levels. Also in the group with methadone maintenance patients we got 85 percent with normal prolactin levels and $15 \%$ with high prolactin levels. But no one in both examination groups had a low prolactin levels (Table 1). Also the results in this study reported that group of street heroin addicts had significantly higher plasma prolactin levels $(\mathrm{p}<0,05)$ then group of patients treated with methadone therapy using t-test for independent samples (Table 2).

\begin{tabular}{lll} 
Prolactin levels & A group (N,\%) & B Group (N,\%) \\
\hline High & $11(55 \%)$ & $3(15 \%)$ \\
Low & $/$ & $/$ \\
Normal & $9(45 \%)$ & $17(85 \%)$ \\
\hline
\end{tabular}

Table 1. Distribution of prolactine serum levels percentage and number of patients in all examination groups.

\begin{tabular}{llccl}
\hline Parametric & A group & B group & Students t-test & p level \\
Prolactin levels & $18,27 \pm 0,72$ & $8,01 \pm 1,23$ & 2,954 & 0,006 \\
\hline
\end{tabular}

Table 2. Statistical significant results of prolactin level between examination groups

\begin{tabular}{lccccc}
\hline Domains of & & \multicolumn{5}{c}{ Scored in domains } \\
SD in IIEF & severe & moderate & mild to moderate & mild & no disfunction \\
\hline Erectile fuction & $/$ & $6(30 \%)$ & $2(10 \%)$ & $3(15 \%)$ & $9(45 \%)$ \\
Orgasmic function & $3(15 \%)$ & $4(20 \%)$ & $2(10 \%)$ & $4(20 \%)$ & $7(35 \%)$ \\
sexual desire & $8(40 \%)$ & $1(5 \%)$ & $1(5 \%)$ & $1(5 \%)$ & $9(45 \%)$ \\
overall satisfaction & $5(25 \%)$ & $4(20 \%)$ & $3(15 \%)$ & $1(5 \%)$ & $7(35 \%)$ \\
\hline
\end{tabular}

Table 3. Distribution on sexual dysfunction (percentage and number of patients in group of methadone maintenance patients

In the group of patients on methadone maintenance therapy $55 \%$ of them have erectile dysfunction, $45 \%$ of them have no erectile dysfunction; $65 \%$ of them have orgasm dysfunction and $35 \%$ of them have no orgasm dysfunction. With dysfunction in sexual desire (libido) in this group we found that in $55 \%$ of causes we got dysfunction in libido, but in $45 \%$ of the subjects we got results with no dysfunction in sexual desire (libido). In patients treated with methadone therapy dysfunction in overall satisfaction was noticed in $65 \%$ of causes and in $35 \%$ of subjects we got no dysfunction in overall satisfaction (Table 3 ). In the group of street heroin addicts $85 \%$ of them have erectile dysfunction and only $15 \%$ of them have no erectile dysfunction. In this group we got that $85 \%$ of them have orgasm dysfunction and $15 \%$ of them do not have orgasm dysfunction. With dysfunction in sexual desire (libido) in this group we got in $80 \%$ of causes, also in $20 \%$ of the subjects we got results with no dysfunction in sexual desire (libido). Dysfunction in overall satisfaction we got in $85 \%$ of causes but in only $15 \%$ of the subjects we got no dysfunction in overall satisfaction (Table 4).

\begin{tabular}{lccccc}
\hline & \multicolumn{2}{c}{ Scored in domains } \\
Domains of IIEF & severe & moderate & mild to moderate & mild & no disfunction. \\
\hline Erectile fuction & $/$ & $12(60 \%)$ & $3(15 \%)$ & $2(10 \%)$ & $3(15 \%)$ \\
Orgasmic function & $7(35 \%)$ & $3(15 \%)$ & $5((25 \%)$ & $2(10 \%)$ & $3(15 \%)$ \\
sexual desire & $7(35 \% 0$ & $2(10 \%)$ & $4(20 \%)$ & $3(15 \%)$ & $4(20 \%)$ \\
overall satisfaction & $7(35 \%)$ & $6(30 \%)$ & $2(10 \%)$ & $2(10 \%)$ & $3(15 \%)$ \\
\hline
\end{tabular}

Table 4. Distribution on sexual dysfunction (percentage and number of patients in heroin addicts group.

In our study we got the results which generally have no statistical significant differences between sexual dysfunction in the examination groups. Results showed that scores of the Questionnaire IIIEF in methadone maintenance patients aren't decreasing significantly in some domains (sexual desire, overall satisfaction $p>0,05$ ) but not in all domains when compare with the group of street heroin addicts. So in the domains of erectile function and orgasm function we got statistical significant results $(\mathrm{p}<$ 0,05) (Table 5).

\begin{tabular}{lrcrc}
\hline Group & erectile function, & orgasm function, & sexual desire & overall satisfaction. \\
A group & $6,90 \pm 1,54$ & $2,84 \pm 0,63$ & $2,96 \pm 0,66$ & $4,38 \pm 0,97$ \\
B group & $8,34 \pm 1,86$ & $3,03 \pm 0,68$ & $3,81 \pm 0,85$ & $4,28 \pm 0,96$ \\
Student's t-test & $\mathrm{p}=0,011$ & $\mathrm{p}=0,033$ & $\mathrm{p}=0,52$ & $\mathrm{p}=0,087$ \\
\hline
\end{tabular}
Table 5 .Statistical significances between 4 domains of sexual dysfunction in examination groups.

\section{DISCUSSION}

This study demonstrated that serum prolactin levels in former heroin addicts on stabile-dose of methadone therapy were significantly lower than the serum prolactin level observed in groups of street heroin addicts. In our study we found that 85 percent of patients in methadone maintenance treatment have a normal prolactin level. This results correlate with another prospective studies which show that hormonal levels return to normal after three or more years of chronic methadone treatment. So, same studies report that stabile dose of methadone therapy always normalize in responsively of several psychological system hypothalamic-pituitary-adrenal, hypothalamic-pituitary-gonad and immune system). Optimum methadone doses tend to stabilize hormonal balances over time $(8,9)$. But in our study we got that $15 \%$ percent of methadone maintenance patients had a high level of serum prolactin, this results compare with another studies who 
reported that after two or more years of chronic high dose of methadone treatment galactorrhoea and another side effects of hyperprolactinemia may persist (10). However some studies reported that in central patient in methadone maintenance treatment increase prolactin levels may persist long time. These results may be modulated by developing the tolerance to dopaminergic effects (tonic-inhibitory controls) of chronic stable dose of methadone treatment (8). But in our study we got result with significant differences between groups of methadone maintenance patients and heroin street addicts $(p<0,05)$. So we got higher levels of prolactin in the group of street heroin addicts and these results correlation with another studies which reported that consequently, the high plasma prolactin levels and sexual dysfunction (SD) as side effects of hyperprolactinemia were found among the patients who entering into addiction treatment. Most substances of abuse and dependency potentially wreak havoc on sexual performance and reproductive function. Thereafter, sexual dysfunction (SD) is apparently common among patents coming into additions treatment. Studies have found that up to $78 \%$ of families and $85 \%$ of males entering methadone maintenance treatment had sexual difficulties while using heroin (11). Also in our study we found that among group of street heroin addicts $85 \%$ of them have erectile dysfunction, $85 \%$ of them have orgasm dysfunction, $80 \%$ have dysfunction of sexual desire and in $85 \%$ of cases we got dysfunction in overall satisfaction. These results may be modulated by develop the tolerance to dopaminergic effects (tonic-inhibitory controls) of chronic stable-dose methadone treatment $(6,7)$. Some studies reported that patients taking methadone had a very high frequency of sexual dysfunction; libido was reduced in $83 \%$ and potency was reduced in $72 \%$ (12). The study of Brown et al also reports that in group of methadone maintenance patients fourteen percent have some sexual dysfunction: erectile dysfunction $(\mathrm{r}=0.24, \mathrm{p}=0.20)$ libido dysfunction $(r=0.30, p=0.003)$ and global dysfunction $(\mathrm{r}-0.26, \mathrm{p}=0.13)$ orgasm dysfunction ( $\mathrm{p}=0.012$ ) (13). After three years or more of chronic high dose methadone treatment in $50 \%$ of patients persistent abnormalities in libido and sexual performance. Ricardo and all in our studies report that among $50 \mathrm{MMT}$ patients $53 \%$ had erectile dysfunction (ED); $26 \%$ had moder- ate to severe erectile dysfunction $(14,15)$. Some studies found that testosterone level remain decreased with SD in around 50$60 \%$ of patients after one years of chronic treatment. However in central patients increase prolactin levels and SD may persist long time (7). Some studies reported very provocative finding of elevation levels of prolactin and decrease levels of testosterone and sexual dysfunction in significant percentage of MMT patients. They return to the normal pattern during variation following detoxification from methadone (16).

In our study we also got results which show that stabile methadone dose return sexual hormone in normal level and decrease sexual dysfunction as side effects of hyperprolactinemia. Among group of patients on methadone maintenance therapy $45 \%$ of them have no erectile dysfunction, $35 \%$ of them have no orgasm dysfunction. Also in $45 \%$ of the subjects we got results with no dysfunction in sexual desire (libido) and in $35 \%$ of subjects we got no dysfunction in overall satisfaction. These results correlation with other prospective studies which found that testosterone level return to normal range with decrease the sexual dysfunction $(8,9)$. Also some prospective, retrospective and special studies reported that after 3 years or more of chronic highdose methadone treatment full stabilization has been achieved (7). So stabile dose of methadone therapy always normalize in responsively of several physiological system; hypothalamic-pituitary-adrenal system. Optimum methadone doses tend to stabilize hormonal balances over time (9). But in our study we also got the results which generally have not statistical significant differences between sexual dysfunction in both examination groups. Results showed that scores of Questionnaire (IIIEF) in methadone maintenance patients decrease significant in some domains as erectile function and orgasm function $(\mathrm{p}<0,05)$ but not in all domains then groups of street heroin addicts. So in the domains of sexual desire and overall satisfaction we got not statistical significant results ( $p>0,05)$.

\section{CONCLUSION}

In our study we got results with higher plasma prolactin level and sexual dysfunction in a group of methadone maintenance patients. So, the patients of methadone treatment attempt to solve this problem many person believe that stimulants such as cocaine or amphetamine enhance sexual function, so patients may resort self-medication with such as stimulants, by reduction doses of prescribed medication, discontinuation of MMT or are dropping out of the treatment.

So replacement treatment with dopamine agonist bromocriptin, and buprenorphine as a opiate antagonist maybe diminished the effects of hyperprolactinemia among the heroin addicts and methadone maintenance patients $(15,16)$. Furthermore patients and staff need education regarding the psychological and psychological impact of drug. So, the patients which enter in methadone maintenance treatment need education of hormonal disorders as a side effects of opioid addiction and their sexual and reproductive health. In this way some condition with hormonal disorders in opiate addicts may be rapidly detect and rapidly affect with some therapeutic models.

\section{REFERENCES}

1. George S, Murali V. Pullickal R. Review of neuroendocrine correlates of chronic opiate misuse: Dysfunction and pathophysiological mechanisms.Addictive Discorders and Their tretamne: 2005; 4(3): 99-109.

2. Bart G, Borg L, Schiuger JH, Green M, Ho A, Kreek MJ. Suppressed prolactin response to dynorphin A113 in methadone maintained versus control subjects. Journal of Pharmacology and experimental therapeutic fast forward. 2003 ; 306: 581-587.

3. Mancini T. Hyperprolactinemia and prolactinomas. Endocrinology and Metabolism. Clinics of North America. 2008; 37: 65.

4. Kulick R, Chaiseha Y, Kang S, Rosenboim L, El Halawani M. The Relative Importance of Vasoactive Intenstinal Peptide Histidine Isoleucine as Physiological regulators of Prolactine in the Domestic Turkey. Gen Comp Endocrinol. 2005; 143(3): 267-273.

5. Matthews DA. Prayer and Spirituality. Rheum Dis. Clin Nort Am. 2000; 2691: 177-187.

6. Caqrter TM. The Effectes of Spiritual Practices on Recovery from Substance Abuse. J Psychiatr Ment Health. 1998; 5(5): 409-413.

7. La Torre D, Falorni A. Pharmacological Cause of Hyperprolactinemia. Ther Clin Risk Manag. 2007; 3(5): 929-951.

8. Kreek MJ, Hartmann N. Chronic Use of Opioids and Effects on Endogenus Opioids and Toxity. Annals New York Academy of Sciences. 51-172.

9. Kreek MJ. Medical Complications in Methadone Patients. Ann NY Acad. Sci. 2002; 311; 110-134.

10. Bennett J, Whale R. Galactorrhoea May be Associated with Methadone Use. BMJ. 2006; 332: 1071.

11. Mahoney MJ, Graci GM. The Meaning and Correlate4s of Spirityality: Suggestions from an Execratory Experts. Death Stud. 2001; 23(6): 521-528.

12. Niclas B, Susane A, Andra S, Klaus W, Detrich K Plasm Testosterone and Sexual Function in Men Receiving Buprenorfine Mmaintence for Opioid Dependence. The Jurnal of Clinical Endocrinology. 2005; 9: 203-206..

13. Brown R, Balausek S, Mudf M, Fleming M. Methadone Maintenance and Male Sexual Dysfunction. J Addict Dis. 2005; 24(2); 91-106.

14. Hallinan R, Byrne A, Tynan Fh, Attia J. Erectile Dysfunction in Men Receiving Methadone and buprenorfine maintenance treatment. J Sex Med. 2008; 5(3): 684-692.

15. Matthews DA. Prayer and Spirituality. Rheum Dosorders. Clinical. North America. 2000; 26(1): 177-187.

16. Shinderman M, Marwell S. Sexual Dysfunction Associated with Methadone Maintenance Treatment with Bromocriptine. Heroin Add.and Rel Clin Probl. 2000; 2(1): 9-14. 